No. 619

December 2019

A time-simultaneous multigrid method for parabolic evolution equations

J. Dünnebacke, S. Turek, P. Zajac, A. Sokolov

ISSN: 2190-1767 


\title{
A time-simultaneous multigrid method for parabolic evolution equations
}

\author{
J. Dünnebacke, S. Turek, P. Zajac, and A. Sokolov
}

\begin{abstract}
We present a time-simultaneous multigrid scheme for parabolic equations that is motivated by blocking multiple time steps together. The resulting method is closely related to multigrid waveform relaxation and is robust with respect to the spatial and temporal grid size and the number of simultaneously computed time steps. We give an intuitive understanding of the convergence behavior and briefly discuss how the theory for multigrid waveform relaxation can be applied in some special cases. Finally, some numerical results for linear and also nonlinear test cases are shown.
\end{abstract}

\section{Motivation}

Modern high performance computing systems feature a growing number of processors and massively parallel co-processors, e.g. GPUs, while the performance of each processor does barely increase or even stagnates. To efficiently use such supercomputers the algorithms have to be massively parallel. The usual time stepping approach to solve time dependent partial differential equations (PDEs) is inherently sequential and does only allow spatial parallelization. If we want to simulate problems with a relatively low number of spatial degrees of freedoms (DOFs), we can only use a certain degree of parallelism, while the number of time steps may be very high due to a long time frame or short time steps. These simulations can not be sped up even if there is more parallel compute power available.

The parallel scalability is limited because the communication between the processes will outweigh the actual computation time, if too many processes are used. It is important to note that usually the main cost of the communication stems from

Jonas Dünnebacke $\cdot$ Stefan Turek · Peter Zajac · Andriy Sokolov

Institute of Applied Mathematics (LS III), TU Dortmund University, Vogelpothsweg 87, D-44227

Dortmund, Germany

e-mail: \{jonas.duennebacke, stefan.turek, peter.zajac, andriy.sokolov\}@ math.tu-dortmund.de 
latency and not from limited bandwidth. If the number of communication operations is reduced by communicating more data at once, the scaling behavior can be improved, so that more processors can be used efficiently in such simulations (see Fig. 3). To achieve this we have to abandon the sequential time stepping.

There already exists a lot of work on time parallel integration. Many methods are based on integrating ODEs parallel in time. The most prominent examples of this group are Parareal [6] and its variants. Another group of time parallel methods is based on solving a global discrete system with multigrid methods. The first parabolic multigrid was developed by Hackbusch [3]. Other representers of such schemes are the one developed by Horton and Vandewalle [4] as well as the recent variant by Gander and Neumüller [2]. The method our approach resembles the most is multigrid waveform relaxation which was first published by Lubich and Ostermann [7]. For a more complete overview on parallel in time methods, we refer to [1].

\section{Time-simultaneous multigrid}

In the following, we propose a multigrid scheme that computes many time steps simultaneously but relies solely on spatial parallelization. Here, we start with a second order parabolic evolution equation

$$
\partial_{t} u(x, t)-\mathcal{L}(t) u(x, t)=f(x, t) \quad(x, t) \in \Omega \times(0, T)
$$

with suitable initial and boundary conditions. $\mathcal{L}(t)$ is a linear elliptic operator for every $t \in(0, T)$. As discretization schemes we consider linear one- or multistep methods in time and finite element (FE) or finite difference (FD) methods in space so that the discrete linear systems of equations (LSE) can be written as

$$
\sum_{m=0}^{M} A_{k, m} \mathbf{u}_{k-m}=\mathbf{f}_{k}, \quad k=1, \ldots, K,
$$

with matrices $A_{k, m} \in \mathbb{R}^{N \times N} . N \in \mathbb{N}$ is the number of spatial degrees of freedom, $K \in \mathbb{N}$ is the number of time steps and $M \in \mathbb{N}$ is the number of steps in the multistep scheme, e.g. $M=1$ for Crank-Nicolson and Euler schemes or $M=R$ for linear R-step methods. Then we can gather the time stepping equations (2) in one global system of the form

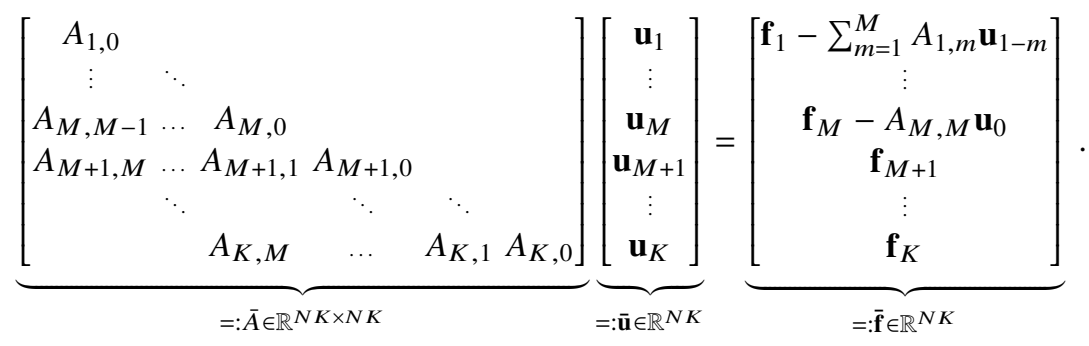


The main idea is to reorder the unknowns from a space-major ordering

$$
\overline{\mathbf{u}}=\left[u_{1,1}, \ldots, u_{1, N}, u_{2,1}, \ldots, u_{2, N}, \ldots, u_{K, 1}, \ldots, u_{K, N}\right]
$$

to a time-major ordering

$$
\mathbf{u}=\left[u_{1,1}, \ldots, u_{K, 1}, u_{1,2}, \ldots, u_{K, 2}, \ldots, u_{1, N}, \ldots, u_{K, N}\right]
$$

where $u_{k, i}=\left(\mathbf{u}_{k}\right)_{i}$ denotes the $i$-th degree of freedom at the $k$-th time step. Reordering the right hand side vector $\overline{\mathbf{f}}$ and the global matrix $\bar{A}$ accordingly leads to the time-blocked system matrix $A$ and the vector $\mathbf{f}$. The Matrix $A$ has the same outer block-structure as the matrices $A_{k, l}$, but each block is a lower triangular $K \times K$ matrix with $M+1$ diagonals.

Now, when we adapt the spatial multigrid method for those systems, we treat each block of the matrix as one entry and use the same transfers and smoothers we would use in a sequential time stepping approach. In our work, we take a Jacobi smoother given by the iteration

$$
\mathbf{u}^{m+1}=\mathbf{u}^{m}+\omega D^{-1}\left(\mathbf{f}-A \mathbf{u}^{m}\right),
$$

where $D$ is the block-diagonal part of the reordered matrix $A$ and $\omega \in \mathbb{R}$ is the damping parameter. As we are formally treating the matrix $A$ as a matrix of blocks, we have to use the complete block-diagonal of $A$ to construct the matrix $D$ instead of only using the main diagonal of $A$. This leads to a block-Jacobi smoother with block dimension $K$. Different smoothers that can be written in the form of eq. (4) with different block-matrices $D$ are applicable in the same manner. The transfer operators are constructed by the same reasoning leading to semi-coarsening in space which means that the transfers in space are applied to each time step independently and the temporal grid stays the same across all levels. With these transfer and smoothing operators the usual multigrid algorithm can be used to solve the LSE incorporating multiple time steps simultaneously.

\subsection{Intuitive explanation for small and large time steps}

We want to give a short intuitive understanding of two special cases that can help to tweak the algorithm in practice. To do this we consider the one dimensional heat equation. In the most simplistic case of central differences as space discretization and an implicit Euler time discretization the discrete scheme is given by

$$
\frac{1}{\tau}\left(u_{k, i}-u_{k-1, i}\right)-\frac{1}{h^{2}}\left(u_{k, i+1}-2 u_{k, i}+u_{k, i-1}\right)=f_{k, i}
$$

with the (fixed) spatial grid size $h$ and the (fixed) time step size $\tau$.

Therefore, the matrix entries belonging to the time derivative are of size $O\left(\tau^{-1}\right)$ whereas the values belonging to discrete Laplace operator are of size $O\left(h^{-2}\right)$. To 
describe the ratio between them we introduce the anisotropy factor $\lambda=\frac{\tau}{h^{2}}$ that is widely used in the convergence analysis of space-time multigrid methods $[2,4,11]$.

As this parameter depends on the temporal and spatial grids, it changes on different levels of the multigrid scheme. Furthermore, it changes locally on each level, if local refinements or space and time dependent diffusion coefficients are used. Consequently the multigrid method should yield fast convergence for all possible $\lambda$.

In the extreme case $\lambda \rightarrow \infty$ the matrix entries belonging to the spatial discretizations prevail. If we ignore the significantly smaller values with a factor of $\tau^{-1}$, each block of the global matrix becomes diagonal, so that the global system consists of $K$ independent $N \times N$ systems. Thus, using the time-blocked multigrid is equivalent to solving each time step with a multigrid scheme on its own. This consideration holds true for all BDF-like time discretizations. Other time discretizations show a similar behavior (see Sect. 3). In the opposite case of $\lambda \searrow 0$ the values of the time derivative dominate and therefore the global system becomes block-diagonal, if the mass matrix is diagonal. A diagonal mass matrix arises naturally in FD discretizations or can be created by using finite elements with mass lumping. With those block-diagonal matrices the undamped block-Jacobi smoother $(\omega=1.0)$ becomes exact and the multigrid solver converges in one step.

An undamped Jacobi smoother is not a suitable smoother generally and we do not want to choose the damping parameter $\omega$ based on $\lambda$ manually. Instead, we suggest to use different smoothers, like the Krylov subspace methods BiCGSTAB [9] or GMRES [8] with the block-diagonal matrix $D$ as a preconditioner. These smoothers yield convergence rates similar to the Jacobi smoothing with comparable effort for large $\lambda$, while they can recover the convergence in one step in the case of $\lambda \searrow 0$ and a diagonal matrix (see Sect. 3).

\subsection{Characteristics of the proposed method}

The time-simultaneous multigrid scheme can be interpreted as a variation of multigrid waveform relaxation (WRMG) (c.f. [7, 5]). Multigrid waveform relaxation methods are based on discretizing the PDE in space and applying a multigrid splitting to the stiffness matrix of the semi-discrete ODE system. When using finite elements such a splitting has to be applied to the mass matrix as well to be able to solve the ODEs that arise in every step of the algorithm independently. These methods are equivalent to the time-simultaneous algorithm if a multigrid splitting with a smoother of the form (4) is used for the mass and stiffness matrices and if the same linear multistep method is used to solve every ODE in the multigrid waveform relaxation scheme. Therefore, we do not provide a more detailed convergence analysis but refer to the literature on WRMG $[11,5]$.

Remark 1 As was shown by Janssen and Vandewalle [5] the time discrete WRMG method for finite elements with a time constant operator $\mathcal{L}$ converges and yields the same asymptotic convergence rates as the traditional multigrid algorithm in the time stepping case, if the coarse grid system matrix $A_{0}$ and the preconditioning matrices 
$D_{l}$ on each level $l$ are regular. Due to the equivalence of both methods this result holds true for the time-simultaneous algorithm.

The spectral radius of the iteration matrix is bounded, but that does not imply that the defect reduction in each iteration is bounded as well, since the iteration matrix is not symmetric. For more complex smoothers like BiCGSTAB and GMRES with a time-blocked preconditioner the result mentioned in remark $1-$ that is based on the spectral radius of the iteration matrix - cannot be applied, because the resulting multigrid iteration is not linear.

The number of necessary floating point operations (FLOPs) in each iteration of the time-simultaneous method with $K$ blocked time steps is still linear in the number of unknowns $N K$. Compared to the time stepping case where a $N \times N$ system is solved by a multigrid method in $K$ time steps, the cost of the grid transfer per iteration and time step is the same. The cost of the defect calculation per iteration and time step is slightly higher in the time-simultaneous case, because the global matrix has a higher bandwidth. The application of the block-diagonal preconditioner $D$ in the smoothing operation (4) also has linear complexity, as each block is a lower triangular matrix with $M$ bands and can be solved by forward substitution.

While the number of required FLOPs of the time-simultaneous method is slightly higher, the number of required communications per multigrid iteration and time step is reduced by a factor of $K^{-1}$, because one multigrid solve yields the solution to $K$ time steps. Consequently, the latency induced time of the communications can be lowered and better parallel scaling is possible. In order to actually achieve this a telescopic multigrid scheme, where on coarser levels fewer processes are used, needs

to be applied. When only a single process is used on the coarse grid, the coarse solve can also be done by time stepping, since no communication is necessary.

The lower triangular solves are inherently sequential, therefore, parallelization in time direction is not trivial. Nevertheless, it is still possible using parallel triangular solvers (c.f. [10]).

To solve non-linear evolution equations we use a time-simultaneous fixed-point or Newton iteration. Using a time stepping scheme we would discretize the equation in time and apply the linearization in each time step, but now we want to solve multiple time steps simultaneously. Therefore, we have to linearize the PDE itself or the global non-linear discrete system.

\section{Numerical results}

In the following, we provide some exemplary results. As a linear test problem we choose the heat equation

$$
\begin{array}{rlrl}
\partial_{t} u-\Delta u & =1+0.1 \sin (t) & (x, t) \in(0,1)^{2} \times(0, T) \\
u(0, t)=u(1, t) & =0 & t \in(0, T) \\
u(x, 0) & =0 & x \in(0,1)
\end{array}
$$


with linear finite elements with mass-lumping as space and a Crank-Nicolson scheme as time discretization. The time-blocked multigrid algorithm uses the F-cycle with one block-Jacobi preconditioned BiCGSTAB pre- and post-smoothing step. For each test 1000 time steps were computed using a different number of blocked time steps. Additionally, we solve the same problem by time stepping and the stationary problem with the same multigrid configuration to create reference results. This was done using spatial grids with grid sizes $h=\frac{1}{32}$ and $h=\frac{1}{128}$. The results are shown in Fig. 1 and 2.

The number of iterations for very small and large time steps behaves as expected. For $\lambda \gg 1$ the number of iterations needed to reduce the global defect by a factor of $10^{-8}$ is independent of the block size and corresponds to the number of iterations that are needed in the stationary test. In the case of $\lambda \ll 1$, the multigrid algorithm converges in one step and in between the number of iteration is at most slightly higher than in the case of large time steps. The only major difference between different block sizes is that the transition area between small and large time steps shifts to smaller time steps if the block size increases.

Comparing the results of different spatial grids shows that the grid size only affects the convergence speed due to its influence on $\lambda$. Other linear multistep methods, higher order finite elements and different test cases show the same qualitative behavior.

To demonstrate the possible benefits of this approach we show the results of a strong scaling test with the same configuration (see Fig. 3) and grid sizes of $h=1 / 256$ and $\tau=0.001$. The method was implemented using the $C++$ based software package $F E A T 3^{1}$ and the tests were executed on the LiDO3 cluster $^{2}$.

With sequential time stepping the best run time is achievable with 32 CPUs and using more processors yields no benefit. Due to the computational overhead, the time-simultaneous approach needs approximately twice the time for low core counts but provides better scaling. Even with a small block size of 20 time steps, more processors can be efficiently used and the run time can be reduced, but with greater block sizes the time-simultaneous scheme scales even better.

To investigate whether this method can be used for non-linear problems we study the behavior of a time-simultaneous linearization with the one-dimensional viscous Burgers' equation

$$
\begin{array}{rr}
\partial_{t} u-\varepsilon \partial_{x x} u+u \partial_{x} u=0 & (x, t) \in(0,1) \times(0, T) \\
u(0, t)=1, u(1, t)=0 & t \in(0, T) \\
u(x, 0)=\max (1-5 x, 0) & x \in(0,1)
\end{array}
$$

with the viscosity $0<\varepsilon \in \mathbb{R}$. Here, we use a FD-discretization with upwind stabilization as discretization in space and Crank-Nicolson in time.

The number of necessary fixed point iteration it to achieve a global defect reduction by $10^{-6}$ depends on the simulated time frame in the case of small diffusion

\footnotetext{
${ }^{1}$ http://www.featflow.de/en/software/feat3.html

2 https://www.lido.tu-dortmund.de/
} 


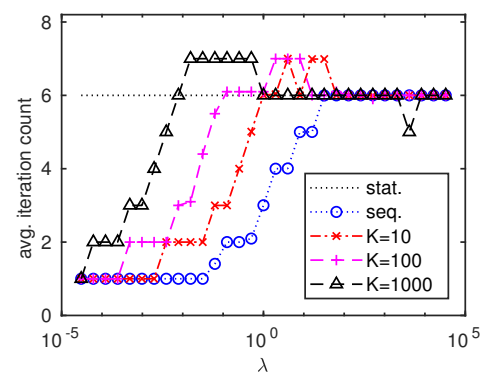

Fig. 1 Number of iterations in the heat eq. test case with different time step sizes and block dimensions, $h=1 / 32$

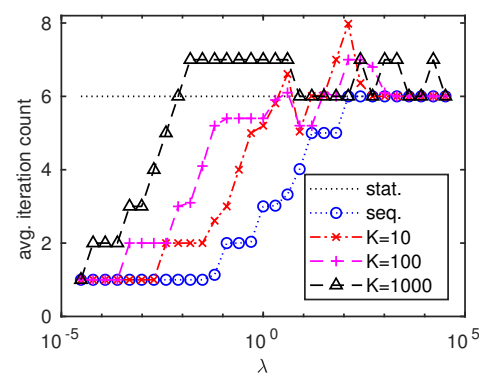

Fig. 2 Number of iterations in the heat eq. test case with different time step sizes and block dimensions, $h=1 / 128$

Fig. 3 Strong scaling test: Solver time for a increasing number of processors, $h=1 / 256$ (65536 spatial elements), $\tau=0.001, T=1$ (1000 time steps)

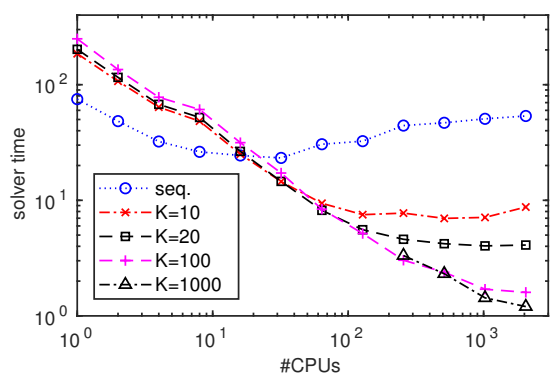

coefficients $\varepsilon$. For example, in the case of $T=1, \tau=0.05$ and $\varepsilon=10^{-3}$ the non-linear solver needs 45 iterations, whereas the averaged number of iterations per time step $i t_{r e f}$ is only 14.25 in the time stepping approach. If the time step size is decreased the number on non-linear iteration in the time stepping case decreases while the global fixed-point iteration does not even manage to converge in 50 steps. Therefore, a time-simultaneous fixed-point iteration is not suitable for the Burgers' equation with a small viscosity.

The Newton scheme provides quadratic convergence if the initial guess is close to the solution. Thus, we compute the solution for the same problem with $2 h, \tau$ and $2 \varepsilon$ and use it as the initial guess for the simulation with the grid sizes $\tau, h$ and the viscosity $\varepsilon$. Using those starting values the number of iterations shows only a slight increase if a longer time frame is calculated simultaneously and in those tests at most 5 iterations are necessary to achieve the desired defect reduction (see Tab. 2).

\section{Conclusion}

We have presented an algebraic approach leading to a time-simultaneous multigrid method that is closely related to multigrid waveform relaxation. The proposed method 


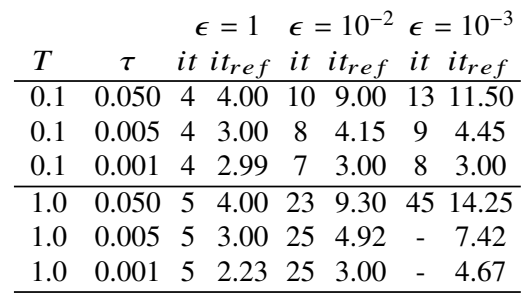

Table 1 Burgers' equation: number of fixedpoint iterations, $h=\frac{1}{2048}$

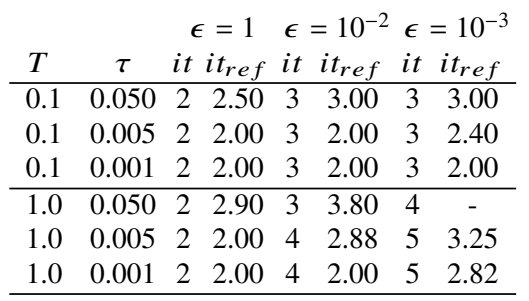

Table 2 Burgers' equation: number of Newton iterations, $h=\frac{1}{2048}$

shows convergence rates that are stable with respect to the number of simultaneous time steps, the grid size and the time step size. The computational cost is slightly higher than in the time stepping case and no parallelization in time direction was done, but the time-simultaneous multigrid method enhances the scalability of the spatial parallelization. The application of this scheme to non-linear equations is also possible by using a time-simultaneous Newton scheme with suitable initial guesses whose choice remains challenging and has to be further examined.

Acknowledgements Calculations have been carried out on the LiDO3 cluster at TU Dortmund. The support by the LiDO3 team at the ITMC at TU Dortmund is gratefully acknowledged.

\section{References}

1. Gander, M.J.: 50 Years of Time Parallel Time Integration. Contrib. Math. Comput. Sci. (2015) doi: 10.1007/978-3-319-23321-5_3

2. Gander, M.J., Neumüller, M.: Analysis of a new space-time parallel multigrid algortihm for parabolic problems. SIAM J. Sci. Comput. 38(4), A2173-A2208 (2016)

3. Hackbusch, W.: Parabolic Multi-grid methods. In R. Glowinski and J.-L. Lions (eds.) Computing Methods in Applied Science and Engineering VI, pp. 189-197, North-Holland (1984)

4. Horton, G., Vandewalle, S.: A space-time multigrid method for parabolic partial differential equations. SIAM J. Sci. Comput. 16(4), 848-864 (1995)

5. Janssen, J., Vandewalle, S.: Multigrid waveform relaxation on spatial finite element meshes: The discrete-time case. SIAM J. Sci. Comput. 17(1), 133-155 (1996)

6. Lions, J-L., Maday, Y., Turinici, G.: Résolution d'EDP par un schéma en temps «pararéel » (2001) doi: 10.1016/S0764-4442(00)01793-6

7. Lubich, C., Ostermann, A.: Multi-grid dynamic iteration for parabolic equations. BIT Numer. Math. 27(2), 216-234 (1987)

8. Saad, Y., Schultz, M.L.: GMRES: A generalized minimal residual algorithm for solving nonsymmetric linear systems. SIAM J. Sci. Stat. Comput. 7(3), 856-869 (1986)

9. Van der Vorst, H.A.: Bi-CGSTAB: A fast and smoothly converging cariant of Bi-CG for the solution of nonsymmetric linear systems. SIAM J. Sci. Stat. Comput. 13(2), 631-644 (1992)

10. Vandewalle, S., Van de Velde, E.: Space-time concurrent multigrid waveform relaxation. Ann. Numer. Math. 1(1-4), 347-363 (1994)

11. Vandewalle, S., Horton, G.: Fourier mode analysis of the multigrid waveform relaxation and time-parallel multigrid methods. Computing (1995) doi: 10.1007/BF02238230 